\title{
The Wnt Signaling Pathway and Related Therapeutic Drugs in Autism Spectrum Disorder
}

\author{
Seung Min Bae ${ }^{1, *}$, ji Yeon Hong ${ }^{2, *}$ \\ ${ }^{1}$ Department of Psychiatry, Gachon University Gil Medical Center, Gachon University College of Medicine, Incheon, ${ }^{2}$ Department of Medicine, \\ Severance Biomedical Science Institute, Yonsei University College of Medicine, Seoul, Korea
}

\begin{abstract}
Autism spectrum disorder (ASD) is a series of neurodevelopmental disorder with a large genetic component. However, the pathogenic genes and molecular mechanisms of ASD have not been clearly defined. Recent technological advancements, such as next-generation sequencing, have led to the identification of certain loci that is responsible for the pathophysiology of ASD. Three functional pathways, such as chromatin remodeling, Wnt signaling and mitochondrial dysfunction are potentially involved in ASD. In this review, we will focus on recent studies of the involvement of Wnt signaling pathway components in ASD pathophysiology and related drugs used in ASD treatment.
\end{abstract}

KEY WORDS: Autism spectrum disorder; Wnt; Beta-catenin; Chromodomain helicase DNA binding protein 8; Neuronal development.

\section{INTRODUCTION}

Autism spectrum disorder (ASD) is a phenotypically heterogeneous group of neurodevelopmental syndrome that is characterized by persistent impairment in reciprocal social communication and social interaction, associated with restricted, repetitive behaviors, as well as showing limited interests or activities. ${ }^{1)}$ ASD is typically apparent during the second year of one's life. In severe cases, deficiencies in developmentally appropriate interest in social interactions may be seen even during the first year of one's life. Verbal and non-verbal problems in so-

Received: July 27, 2017 / Revised: September 27, 2017

Accepted: September 28, 2017

Address for correspondence: Seung Min Bae, MD

Department of Psychiatry, Gachon University Gil Medical Center,

Gachon University College of Medicine, 774 Bun-gil,

Namdongdae-ro, Namdong-gu, Incheon 21565, Korea

Tel: +82-32-1577-2299, Fax: +82-32-472-3396

E-mail: manitch@hanmail.net

ORCID: https://orcid.org/0000-0003-3732-6824

Ji Yeon Hong, PhD

Department of Medicine, Severance Biomedical Science Institute,

Yonsei University College of Medicine, 50 Yonsei-ro,

Seodaemun-gu, Seoul 03722, Korea

Tel: +82-2-337-9972, Fax: +82-0504-200-0318

E-mail: mire1390@gmail.com

ORCID: https://orcid.org/0000-0002-8599-220X

*These authors contributed equally to this work. cial communication have varying manifestations depending on the severity of the disorder as well as several other factors, including appropriate treatment history and environmental support.

Despite the serious characteristics of this disorder, unfortunately, only early detection and special education are considered appropriate treatments until now. Earlier and more appropriate diagnosis and treatment reduce the severity of symptoms and improve adaptability to social life, providing higher quality of life to ASD individuals and their families. Although this earlier diagnosis is critically important due to the severity of this disorder, diagnosis of ASD is not simple and cannot be made by clearcut diagnostic tools. Neuroanatomical changes visualized by magnetic resonance imaging (MRI), computed tomography $(\mathrm{CT})$ and postmortem analysis do not provide clear diagnostic evidence. ${ }^{2)}$ Genetic heritability estimates for ASD have ranged from $37 \%$ to $92 \%$ in twin studies. As many as $15 \%$ of cases of ASD appear to be associated with a genetic mutation. ${ }^{1)}$ Some genetic disorders, such as Rett syndrome, fragile X syndrome, and Down syndrome are related to ASD. However, the cause of ASD is often unknown and ASD is considered a heterogeneous disease entity, which has difficulties with early diagnosis.

In addition to the trouble of early diagnosis, only two

(ㄷ) This is an Open-Access article distributed under the terms of the Creative Commons Attribution Non-Commercial License (http://creativecommons.org/licenses/by-nc/4.0) which permits unrestricted non-commercial use, distribution, and reproduction in any medium, provided the original work is properly cited. 
medications are approved to treat one of core symptoms of autism. However, none of treating options for autism work well for every individual, since the autism is not a monogenic disorder. Recent advances in next-generation sequencing have provided new molecular insights into the etiology of ASD. Intriguingly, multiple mutations in Wnt signaling components have been identified from autistic individuals. Thus, this suggests the possibility that mutations in Wnt signaling could be one of a major cause of this mental illness and understanding of these mechanisms might open the possibility of early diagnosis and new treatment era for ASD. In this review, we will discuss mutations in Wnt pathway components in individuals with ASD. Here, we will focus on recent progress in human genetic studies, since animals' and cellular data were already covered by other reviews. In addition, we will cover potential medications which are related to the Wnt pathway that may ameliorate these defects, thus allowing individuals with ASD to better adapt to our society.

\section{MOLECULAR SIGNATURES OF ASD}

As described above, although ASD is a pervasive developmental disorder, the etiology of ASD is poorly understood. Initial population-based studies have failed to identify a single ASD-associated gene and some risk variants, despite the strong heritability of this disease.

Since 2012, number of mutations and gene sets has been identified by multiple approaches, including nextgeneration sequencing, clinically used medications and mouse models. ${ }^{3,4)}$ Based on the clinical heterogeneity of ASD as we described above, it is not surprising that multiple genetic mutations exist. Exome sequencing studies have revealed that many single nucleotide polymorphisms (SNPs) presents in ASD individuals and that these genes converge onto the Wnt pathway (Fig. 1). Furthermore, mounting evidence indicates that mutations in Wnt pathway-related genes contribute to ASD. ${ }^{4)}$

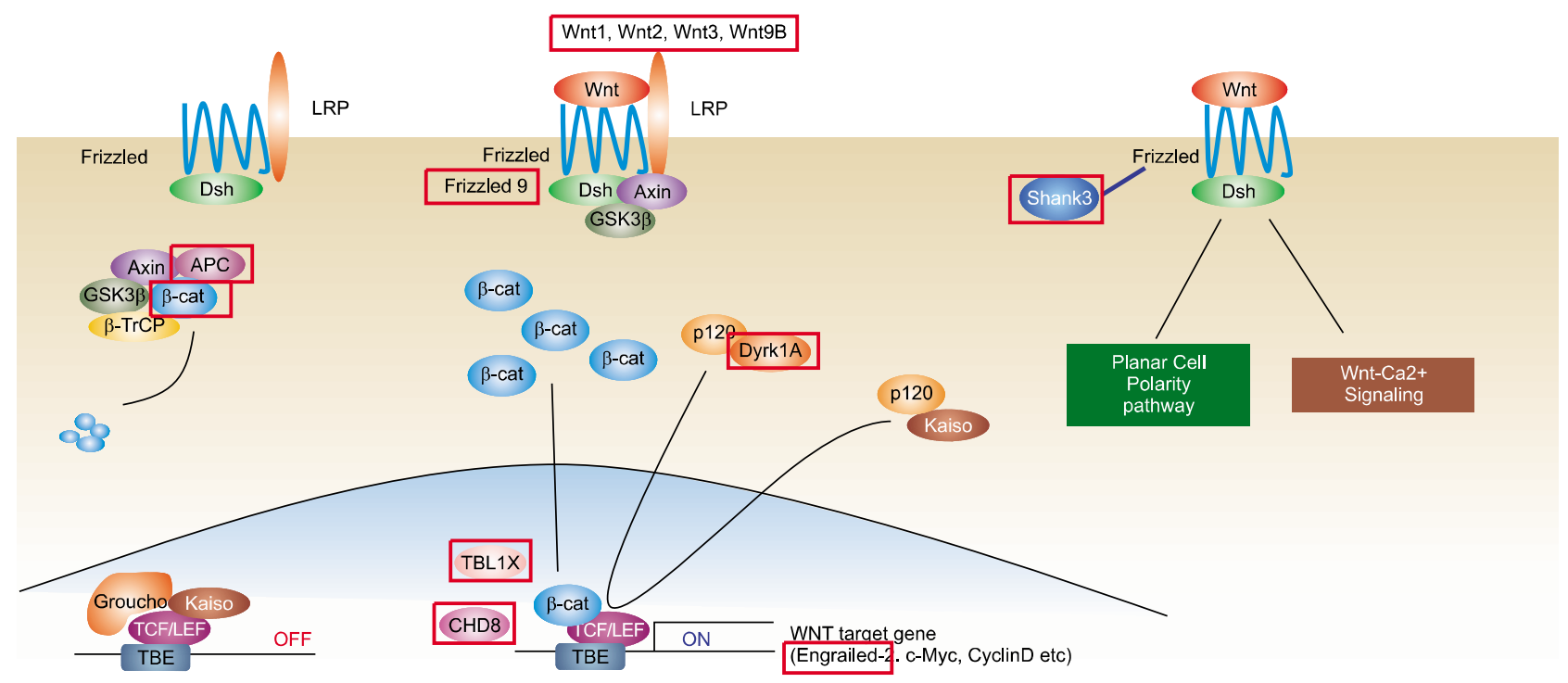

Fig. 1. Mechanisms regulating the canonical and non-canonical Wnt pathway. In the absence of Wnt ligands, $\beta$-catenin is degraded by the destruction complex. $\beta$-catenin is stabilized by the association of Wnt ligands with receptors such as Frizzled and lipoprotein receptor-related proteins 5 and 6 (LRP5/6). Stabilized $\beta$-catenin can enter the nucleus and activates Wnt target genes including Engrailed-2. Red boxes indicate genes mutated in individuals with autism spectrum disorder, which are identified from next generation sequencings.

Dsh, dishevelled; Shank3, SH3 and multiple ankyrin repeat domain 3; GSK, glycogen synthase kinase; APC, adenomatosis polyposis coli; Dyrk1A, dual specificity tyrosine-phosphorylation-regulated kinase 1A; TBL1X, transducin beta like 1X-linked; CHD8, chromodomain-helicase-DNAbinding protein 8; TCF/LEF, T-cell factor/lymphoid enhancer factor; TBE, TCF-binding element; LRP, low density lipoprotein receptor-related protein. 


\section{The Canonical Wnt Signaling Pathway in Patients with ASD}

The Wnt signaling pathway is one of the major mechanisms during embryonic development and tumorigenesis. ${ }^{5-8)}$ Wnt proteins are secreted as cysteine-rich glycolipoproteins that act as ligands. Upon stimulation of receptors, such as Frizzled, by Wnt protein association, the Wnt signaling pathway govern multiple processes, including embryonic development and tissue homeostasis. The Wnt signaling pathway is classified into the "canonical" ( $\beta$-catenin-mediated) and "non-canonical" ( $\beta$-catenin-independent) pathways. ${ }^{5)}$ The non-canonical Wnt signaling pathway consists of calcium signaling and planar cell polarity pathways. In contrast, canonical Wnt signaling is mediated by $\beta$-catenin and plays important roles in cell proliferation, differentiation and migration during development. ${ }^{9)}$ Without Wnt stimulation, $\beta$-catenin is constitutively degraded by the destruction complex, such as glycogen synthase kinase 3 beta (GSK3 $\beta$ ), casein kinase 1 alpha $(C K 1 \alpha)$, axin and adenomatous polyposis coli (APC). Phosphorylation by GSK3 $\beta$ and CK1 $\alpha$ facilitates the degradation of $\beta$-catenin via the ubiquitin-mediated proteasome pathway. When Wnt signaling is activated, Wnt ligands associates with Frizzled and co-receptors, low-density lipoprotein receptor-related proteins 5 and 6 (LRP5/6). Upon Wnt stimulation, cytoplasmic Dishevelled relays signals to the destruction complex components, such as GSK3 $\beta$ and Axin. GSK3 $\beta$ and Axin are then recruited to the membrane, and $\beta$-catenin segregates from the destruction complex. Stabilized $\beta$-catenin can enter the nucleus and displace Goucho/transducin-like enhancer of split (TLE) repressors from T-cell factor/lymphoid enhancer factor (TCF/LEF) transcription factors to activate multiple $\mathrm{Wnt}$ target genes. ${ }^{10)}$ There are $19 \mathrm{Wnt}$ genes and 10 Frizzled genes, conferring substantial complexity to the Wnt pathway.

Genome-wide screens have shown that ASD is associated with Wnt components through mutations in the genes encoding $\mathrm{Wnt} 1,{ }^{11)} \mathrm{Wnt} 2,{ }^{12)}$ phosphatase protein tyrosine phosphatase receptor type Z1 (PTPRZ1), ${ }^{13)}$ Engrailed2, ${ }^{14,15)}$ APC, ${ }^{16)} \beta$-catenin ${ }^{17)}$ and chromodomain helicase DNA binding protein 8 (CHD8) ${ }^{17-21)}$ (Fig. 1). APC is part of the destruction complex in the Wnt pathway, serving as a negative modulator and scaffold protein of $\beta$-catenin for ubiquitin-mediated proteosomal degradation.
Altered Wnt signaling via stabilized $\beta$-catenin has been identified in multiple cancers, and mutations in APC are frequently observed in colon cancer. Recent evidence has shown that the $A P C$ gene is related to the risk of neurodevelopmental brain disorders. ${ }^{16)}$ Next-generation sequencing has also shown that single nucleotide variants of Wnt1 and Wnt2 are associated with ASD, again suggesting that altered Wnt signaling contributes to ASD susceptibility. ${ }^{11,12)}$ Based upon genome-wide screening data, de novo mutations in CHD8 are strongly associated with autism. Mounting evidences using cell lines and mouse models support their strong correlations. Recent study revealed that CHD8 knockdown in mouse brain leads to defective neural progenitor proliferation and differentiation, followed by abnormal neural morphology. ${ }^{22)}$ This report also showed that CHD8 knockdown disrupts the expression of $\mathrm{Wnt}$ target genes and ectopic expression of stabilized form of $\beta$-catenin rescued the defective phenotype of embryonic mouse brain and N2a cells.

The CTNNB1 gene, which encodes $\beta$-catenin, is a main modulator of the canonical Wnt signaling pathway and is linked to sporadic ASD and intellectual disability. Nonsense and missense mutations in CTNNB1 have been identified in patients with intellectual disability and ASD. ${ }^{17)}$ Furthermore, multiple knockout mice have been generated, and $C T N N B 1$ has shown to be a fundamental component in brain development. ${ }^{23)}$ Conditional knockout mice lacking the expression of $C T N N B 1$ in the paravalbumin interneurons displayed impaired object recognition and social interactions as well as elevated repetitive behaviors. Cellular models also support that CTNNB1 is an important modulator of brain development, especially neuronal differentiation and cortical development. One report provides a model that $\mathrm{N}$-cadherin, which is abundantly expression in neuronal cells, regulates $\beta$-catenin via Akt. ${ }^{24)}$

Recently, genome-wide association studies of the $X$ chromosome have shown that transducin beta-like 1X-linked (TBL1X), a novel component of the Wnt pathway, is related with ASD. ${ }^{25)}$ Moreover, Chung et al. ${ }^{25)}$ found intronic SNPs in TBL1X and other studies identified deletions in the Xp22.2 to Xp22.3 region, which includes TBL1X, in autistic girls and women. Because TBL1X interacts with $\beta$-catenin and binds to the promoter of Wnt target genes, it is plausible that the association between TBL1X and ASD could be mediated by the Wnt pathway. 
Consistent with this, certain Wnt target genes have also shown to be associated with ASD. Two intronic SNPs in Engrailed2 were found to be strongly correlated with ASD. ${ }^{14,15)}$

\section{p120-catenin Family Members in the Canonical Wnt Pathway}

p120-catenin, structurally similar to $\beta$-catenin, is also involved in the canonical Wnt signaling pathway and is modulated by the destruction complex, which includes GSK3 $\beta$, Axin and APC. ${ }^{26)}$ P120-catenin family members include p120-catenin itself, $\delta$-catenin, ARVCF (Armadillo repeat protein deleted in velo-cardio-facial syndrome) and p0071. P120-catenin activates Wnt target genes by relieving Kaiso-mediated repression. ${ }^{27)}$ In addition, activation of Wnt signaling stabilizes p120-catenin in a manner similar to that of $\beta$-catenin. Intriguingly, Dryk1A has been shown to regulate the stability of p120-catenin and modulates Wnt target genes through the p120-catenin/Kaiso pathway. ${ }^{28)}$ Dyrk1A has also shown to be associated with ASD from sequencing studies, and mutations in this gene are among the top five ASD- and ID-related genes, ${ }^{17,21)}$ suggesting that the Dyrk1A/p120-catenin pathway may be related to ASD.

p120-catenin family members are also involved the point at which Wnt signaling meets small GTPases, such as RhoA and Rac. $\delta$-catenin, a member of the p120-catenin family, is known to be particularly vulnerable to genetic variations in some human diseases. For example, $\delta$-catenin harbors significantly more deleterious missense mutations and copy number variations in autistic patients compared to controls. ${ }^{29,30)}$ Interestingly, evaluation of these variations has been further implicated in Wnt signaling and functional tests have shown that these variations result in loss of function. Thus, Wnt/p120/Dyrk1A pathway may provide an interesting perspective in the mechanisms regulating ASD and therapeutic targets.

\section{The Non-canonical Wnt Signaling Pathway in ASD}

Limited information on the relationship between components of the non-canonical Wnt signaling and ASD is available. Non-canonical Wnt signaling includes the Wnt/PCP pathway and the $\mathrm{Wnt} / \mathrm{Ca}^{2+}$ pathway. Disruption of the non-canonical Wnt gene, PRICKLE2 (planar cell polarity protein2), results in autism-like behaviors in mice. ${ }^{31)}$ Additionally, $\mathrm{SH} 3$ and multiple ankyrin repeat domains (SHANK), a newly identified protein that modulates non-canonical Wnt signaling, ${ }^{32)}$ has shown to be a cause of ASD in recent studies of human genetics. ${ }^{33,34)}$ However, there is no concrete relationship between the non-canonical Wnt pathway and mental illnesses, including ASD.

\section{Therapeutic Effects of Drugs to Treat ASD May Be Related to Canonical Wnt Signaling}

As described above, there are currently no known cures for ASD. There are only two drug, i.e., risperidone and aripiprazole, which have been approved by the US Food and Drug Administration for the treatment of ASD's core symptom. However, both of them are not intended for the complete cure, but only to alleviate symptoms such as irritability in ASD patients. Thus, the development of new therapeutic drugs to treat individuals affected by ASD is urgently required. Some antipsychotics (e.g., risperidone and aripiprazole), mood stabilizers (e.g., lithium) and antidepressants are prescribed to correct the aggressive behaviors associated with ASD. ${ }^{2)}$

Given the relationship between Wnt signaling and ASD illustrated above, recent studies have evaluated whether medications used to treat autism affect the Wnt signaling pathway. Interestingly, haloperidol and clozapine (an antipsychotic medication), ${ }^{35,36)}$ fluoxetine (a selective serotonin reuptake inhibitor) ${ }^{37,38)}$ and Ritalin (methyphenidate) ${ }^{39)}$ have shown to modulate Wnt signaling. Haloperidol is known to transiently increase the phosphorylation of Akt, followed by inhibition of GSK3 $\beta{ }^{40)}$ Another report also showed that haloperidol promotes the expression of certain Wnt components, such as Wnt-5a, Dishevelled-3 and $\beta$-catenin. Haloperidol may relay signals through D2 dopamine receptors, which are associated with Dishevelled-3. ${ }^{41)}$ Fluoxetine has also been shown to be involved in Wnt signaling, as indicated by mouse studies. Fluoxetine downregulates $m i R-16$, thereby promoting the expression of Wnt2, a brain-derived neurotrophic factor (BDNF). ${ }^{38)}$ Ritalin is identified to regulate Akt and GSK3, suggesting potential involvement in the Wnt signaling pathway. ${ }^{39)}$ Although we must exercise caution when approaching medical treatments related to the Wnt pathway, it is intriguing that some medications used to treat autism directly or indirectly modulate Wnt components, including GSK3. Lithium is also used to alleviate aggression to treat individuals with autism and likely be related to Wnt sig- 
naling, lithium may modulate various signal transduction pathways beyond neurotransmitter receptors (includes second messengers) and modulate $G$ proteins, regulate the expression of growth factors, and influence neuronal plasticity through interaction with downstream signal transduction cascades, including inhibition of GSK3. These effects on GSK3 could explain the involvement of lithium in the Wnt signaling pathway. Further studies are needed to determine the involvement of this mechanism and compare the efficacy of lithium in individuals with and without Wnt signaling abnormalities. Thus, the regulation of Wnt signaling with medication could be an important and plausible treatment strategy.

Given that the aberrant regulation of the Wnt pathway is one of the prevalent molecular pathology in cancer biology, autism and cancer share risk genes, pathways and drug targets. ${ }^{42)}$ Thus, repurposing cancer drugs, especially targeting the Wnt pathway, could be considered in clinical trials to treat autistic individuals. It will largely reduce time frame, cost and improve success rates. Another significant advantage of drug repurposing over traditional drug development is that detained information including toxicity and stability test is available and the risk of failure due to adverse toxicity can be reduced. Multiple new drugs targeting the Wnt pathway are currently being evaluated in preclinical, phase I and phase II trials to treat cancer patients. However, none of those drugs are approved to treat neurological diseases. Until now, there are some attempts to repurpose cancer drugs to treat neurological diseases. One intriguing example is Tideglusib (ClinicalTrials.gov identifier: NCT02586935), which is developed as GSK3 $\beta$ inhibitor of the Wnt pathway, but failed in cancer trials. This drug is now clinical Trial phase 2 to treat adolescents with ASD. Another example is saracatinib (AZD0530, ClinicalTrials.gov identifier: NCT00397878), which is now in Phase2a trail to treat Alzheimer's disease. Therefore, repositioning cancer drugs related to the $\mathrm{Wnt}$ pathway components to treat ASD would provide new candidate therapies quickly and improve quality of life of autistic individuals. ${ }^{43}$

\section{CONCLUSION}

Efforts in ASD studies have focused on identifying reliable biomarkers based on multiple approaches, including genetics and neuroimaging. Despite major advancements in studies of neuronal development, there are no specific biomarkers of ASD to support routine clinical use. Neuroimaging studies employing CT and MRI are also controversial. Since early treatment of ASD may increase the efficacy of treatment, finding a reliable biomarker for detecting ASD when illness is not clear yet, could eventually help to apply more accurate and appropriate treatment for patient.

Given that Wnt target genes are thought to be involved in ASD, genetic clusters of Wnt pathway genes could reflect the possibility of ASD and provide an effective approach for categorizing ASD based on their pathophysiology and gene expression criteria. This will contribute to early detection of the ASD phenotype.

Furthermore, we can also search and evaluate certain drugs that are already known to regulate Wnt pathway and assess whether such drugs may be effective for the treatment of ASD based on genetic studies and biomarkers. These studies are expected to reveal important connections between basic neuroscience and clinical studies of mental illness and improve our mental health.

\section{- Acknowledgments}

This work was supported by a grant from the National Research Foundation of Korea (NRF) funded by the Korean government (Ministry of Science, ICT \& Future Planning; NRF-2015R1C1A1A02036506) to J.Y.H. We also thank to Yoon Young Kim and Seungjin Bai for their help and support. The authors declare that they have no conflicts of interest.

\section{REFERENCES}

1. American Psychiatric Association. Neurodevelopmental disorder. Section II. Diagnostic criteria and codes. In: American Psychiatric Association. the Diagnostic and Statistical Manual of Mental Disorders 5th edition: DSM-5. Washington, D.C.:American Psychiatric Publishing;2013.

2. Sadock BJ, Sadock VA, Ruiz P. Kaplan and Sadock's synopsis of psychiatry: behavioral science/clinical psychiatry. 11th ed. Philadelphia:Wolters Kluwer;2015.

3. Caracci MO, Ávila ME, De Ferrari GV. Synaptic Wnt/GSK3 $\beta$ signaling hub in autism. Neural Plast 2016;2016:9603751.

4. Mulligan KA, Cheyette BN. Neurodevelopmental perspectives on Wnt signaling in psychiatry. Mol Neuropsychiatry 2017:2:219-246.

5. Loh KM, van Amerongen R, Nusse R. Generating cellular diversity and spatial form: Wnt signaling and the evolution of multicellular animals. Dev Cell 2016;38:643-655. 
6. Clevers H, Loh KM, Nusse R. Stem cell signaling. An integral program for tissue renewal and regeneration: Wnt signaling and stem cell control. Science 2014;346:1248012.

7. Anastas JN, Moon RT. WNT signalling pathways as therapeutic targets in cancer. Nat Rev Cancer 2013;13:11-26.

8. Muneer A. Wnt and GSK3 signaling pathways in bipolar disorder: clinical and therapeutic implications. Clin Psychopharmacol Neurosci 2017;15:100-114.

9. Nusse R, Clevers H. Wnt/ $\beta$-catenin signaling, disease, and emerging therapeutic modalities. Cell 2017;169:985-999.

10. Daniels DL, Weis WI. Beta-catenin directly displaces Groucho/TLE repressors from Tcf/Lef in Wnt-mediated transcription activation. Nat Struct Mol Biol 2005; 12:364-371.

11. Martin PM, Yang X, Robin N, Lam E, Rabinowitz JS, Erdman $\mathrm{CA}$, et al. A rare WNT1 missense variant overrepresented in ASD leads to increased Wnt signal pathway activation. Trans/ Psychiatry 2013;3:e301.

12. Wassink TH, Piven J, Vieland VJ, Huang J, Swiderski RE, Pietila J, et al. Evidence supporting WNT2 as an autism susceptibility gene. Am J Med Genet 2001;105:406-413.

13. Bonora E, Lamb JA, Barnby G, Sykes N, Moberly T, Beyer KS, et al. Mutation screening and association analysis of six candidate genes for autism on chromosome 7q. Eur I Hum Genet 2005; 13:198-207.

14. Benayed R, Gharani N, Rossman I, Mancuso V, Lazar G, Kamdar S, et al. Support for the homeobox transcription factor gene ENGRAILED 2 as an autism spectrum disorder susceptibility locus. Am J Hum Genet 2005;77:851-868.

15. Gharani N, Benayed R, Mancuso V, Brzustowicz LM, Millonig $\mathrm{JH}$. Association of the homeobox transcription factor, ENGRAILED 2, 3, with autism spectrum disorder. Mol Psychiatry 2004;9:474-484.

16. Mohn JL, Alexander J, Pirone A, Palka CD, Lee SY, Mebane L, et al. Adenomatous polyposis coli protein deletion leads to cognitive and autism-like disabilities. Mol Psychiatry 2014; 19:1133-1142.

17. Krumm N, O'Roak BJ, Shendure J, Eichler EE. A de novo convergence of autism genetics and molecular neuroscience. Trends Neurosci 2014;37:95-105.

18. Barnard RA, Pomaville MB, O'Roak BJ. Mutations and mode/ing of the chromatin remodeler CHD8 define an emerging autism etiology. Front Neurosci 2015;9:477.

19. Sanders SJ. First glimpses of the neurobiology of autism spectrum disorder. Curr Opin Genet Dev 2015;33:80-92.

20. O’Roak BJ, Vives L, Girirajan S, Karakoc E, Krumm N, Coe BP, et al. Sporadic autism exomes reveal a highly interconnected protein network of de novo mutations. Nature 2012;485: 246-250.

21. O’Roak BJ, Vives L, Fu W, Egertson JD, Stanaway IB, Phelps $\mathrm{IG}$, et al. Multiplex targeted sequencing identifies recurrently mutated genes in autism spectrum disorders. Science 2012; 338:1619-1622.

22. Durak O, Gao F, Kaeser-Woo YJ, Rueda R, Martorell AJ, Nott
A, et al. Chd8 mediates cortical neurogenesis via transcriptional regulation of cell cycle and Wnt signaling. Nat Neurosci 2016;19:1477-1488.

23. Dong F, Jiang J, McSweeney C, Zou D, Liu L, Mao Y. Deletion of CTNNB1 in inhibitory circuitry contributes to autism-associated behavioral defects. Hum Mol Genet 2016;25:2738-2751.

24. Zhang J, Shemezis JR, McQuinn ER, Wang J, Sverdlov M, Chenn A. AKT activation by $\mathrm{N}$-cadherin regulates beta-catenin signaling and neuronal differentiation during cortical development. Neural Dev 2013;8:7.

25. Chung RH, Ma D, Wang K, Hedges DJ, Jaworski JM, Gilbert $\mathrm{JR}$, et al. An X chromosome-wide association study in autism families identifies TBL $1 X$ as a novel autism spectrum disorder candidate gene in males. Mol Autism 2011;2:18.

26. Hong JY, Park JI, Cho K, Gu D, Ji H, Artandi SE, et al. Shared molecular mechanisms regulate multiple catenin proteins: canonical Wnt signals and components modulate p120-catenin isoform-1 and additional p120 subfamily members. J Cell Sci 2010;123:4351-4365.

27. Park JI, Kim SW, Lyons JP, Ji H, Nguyen TT, Cho K, et al. Kaiso/p120-catenin and TCF/beta-catenin complexes coordinately regulate canonical Wnt gene targets. Dev Cell 2005; 8:843-854.

28. Hong JY, Park JI, Lee M, Muñoz WA, Miller RK, Ji H, et al. Down's-syndrome-related kinase Dyrk1A modulates the p120-catenin-Kaiso trajectory of the Wnt signaling pathway. J Cell Sci 2012;125:561-569.

29. Lu Q, Aguilar BJ, Li M, Jiang Y, Chen YH. Genetic alterations of $\delta$-catenin/NPRAP/Neurojungin (CTNND2): functional implications in complex human diseases. Hum Genet 2016; 135:1107-1116.

30. Turner TN, Sharma K, Oh EC, Liu YP, Collins RL, Sosa MX, et al. Loss of $\delta$-catenin function in severe autism. Nature 2015;520:51-56.

31. Sowers LP, Loo L, Wu Y, Campbell E, Ulrich JD, Wu S, et al. Disruption of the non-canonical Wnt gene PRICKLE2 leads to autism-like behaviors with evidence for hippocampal synaptic dysfunction. Mol Psychiatry 2013;18:1077-1089.

32. Harris KP, Akbergenova Y, Cho RW, Baas-Thomas MS, Littleton JT. Shank modulates postsynaptic wnt signaling to regulate synaptic development. J Neurosci 2016;36:5820-5832.

33. Leblond CS, Nava C, Polge A, Gauthier J, Huguet G, Lumbroso S, et al. Meta-analysis of SHANK mutations in autism spectrum disorders: a gradient of severity in cognitive impairments. PLoS Genet 2014;10:e1004580.

34. Gauthier J, Spiegelman D, Piton A, Lafrenière RG, Laurent S, St-Onge J, et al. Novel de novo SHANK3 mutation in autistic patients. Am J Med Genet B Neuropsychiatr Genet 2009; 150B:421-424.

35. Kwan V, Unda BK, Singh KK. Wnt signaling networks in autism spectrum disorder and intellectual disability. J Neurodev Disord 2016;8:45.

36. Amamou B, Salah WB, Mhalla A, Benzarti N, Elloumi $H$, Zaafrane $\mathrm{F}$, et al. Use of clozapine for borderline personality 
disorder: a case report. Clin Psychopharmacol Neurosci 2016; 14:226-228.

37. Zhou WJ, Xu N, Kong L, Sun SC, Xu XF, Jia MZ, et al. The antidepressant roles of Wnt2 and Wnt3 in stress-induced depression-like behaviors. Trans/ Psychiatry 2016;6:e892.

38. Launay JM, Mouillet-Richard S, Baudry A, Pietri M, Kellermann O. Raphe-mediated signals control the hippocampal response to SRI antidepressants via miR-16. Trans/ Psychiatry 2011;1:e56.

39. Mines MA, Jope RS. Brain region differences in regulation of Akt and GSK3 by chronic stimulant administration in mice. Cell Signal 2012;24:1398-1405.

40. Roh MS, Seo MS, Kim Y, Kim SH, Jeon WJ, Ahn YM, et al.
Haloperidol and clozapine differentially regulate signals upstream of glycogen synthase kinase 3 in the rat frontal cortex. Exp Mol Med 2007; 39:353-360.

41. Sutton LP, Honardoust D, Mouyal J, Rajakumar N, Rushlow WJ. Activation of the canonical Wnt pathway by the antipsychotics haloperidol and clozapine involves dishevelled-3. J Neurochem 2007;102:153-169.

42. Crawley JN, Heyer WD, LaSalle JM. Autism and cancer share risk genes, pathways and drug targets. Trends Genet 2016; 32:139-146.

43. Kahn M. Can we safely target the WNT pathway? Nat Rev Drug Discov 2014; 13:513-532. 